Gerontology 1988;34(suppl. 1):45

\title{
Author Subject Indexes
}

Bellamy, N. $16 \quad$ Aging 11

Calin, A. 1,42 Arthritis care 42

Hughes, G. 27 Cost/benefit ratio 42

Lequesne, M. $33 \quad$ Elderly, arthritis 16

Svanborg, A. 11 Epidemiology 2

Valkenburg, H.A. 2 Geriatric 11

- $\quad$ conditions 2

Health expenditures 2

Joint function 11

Kidney function 27

Life expectancy 2

Liver function 27

Locomotor 11

Nonsteroidal antiinflammatory drugs 33

- $\quad$ drugs 27

Osteoarthritis 11

Rheumatoid arthritis 11, 33

Side effects, nonsteroidal antiinflammatory

drugs 33 Steroid 27 Treatment arthritis 16

risks 33 